\title{
Estimation, Modeling, and Simulation of Patterned Growth in Extreme Environments
}

\author{
B. Strader, K.E. Schubert, M. Quintana, E. Gomez, J. Curnutt, and P. Boston
}

\begin{abstract}
In the search for life on Mars and other extraterrestrial bodies or in our attempts to identify biological traces in the most ancient rock record of Earth, one of the biggest problems facing us is how to recognize life or the remains of ancient life in a context very different from our planet's modern biological examples. Specific chemistries or biological properties may well be inapplicable to extraterrestrial conditions or ancient Earth environments. Thus, we need to develop an arsenal of techniques that are of broader applicability. The notion of patterning created in some fashion by biological processes and properties may provide such a generalized property of biological systems no matter what the incidentals of chemistry or environmental conditions. One approach to recognizing these kinds of patterns is to look at apparently organized arrangements created and left by life in extreme environments here on Earth, especially at various spatial scales, different geologies, and biogeochemical circumstances.
\end{abstract}

\section{Introduction}

A key aspect of planning a space mission is to set scientific mission objectives with the ability to adapt them based on observations and mission situations. The search for extraterrestrial life is a major scientific objective, but the exact nature of that life and how to confirm it constitutes a major debate [9]. A further problem in the search for extraterrestrial life is how to select specific areas that we want to investigate more intensively? Photo surveys, geology, and knowledge of biology here on Earth can take us to a likely general area, but, unlike a dynamic process like that which

B. Strader, K.E. Schubert, M. Quintana, E. Gomez, J. Curnutt

California State University, San Bernardino, San Bernardino, CA 92407 e-mail:

\{bstrader,schubert,quintanm,ernesto\}@csusb.edu, jcurnutt@r2labs.org

P. Boston

New Mexico Tech, Socorro, NM 87801 e-mail: pboston@nmt.edu 
produced the Martian dust devils [2], subtle biological clues have no motion to point us in the right direction.

We use life in extreme environments on Earth as analogs for the kinds of life that we could encounter in space [22]. We propose using biopatterns found in some of these analogs to create a series of templates that could be used to indicate areas potentially worthy of deeper investigation. In resource-limited environments (often the case with extreme environments), organisms grow in patterns that are self-enforcing and exhibit hysteresis $[1,21,14]$ which could be used to recognize them and their fossil remains at a distance. Particularly on Mars, as the environment apparently became less hospitable over geologic time, extremophiles similar to Earth's were likely the last to survive, and should be the easiest to find at least in environments protected from the weathering effects of the harsh surface climate. Obviously, caves would provide such protection [6]. Among the techniques that have been used to model these patterns are evolutionarily stable strategies in game theory and differential equations $[8,15,21,29,14]$.

While good results have been generated using differential equations, they require tuning of the parameters and experience in mathematical and numerical techniques to obtain valid results. In this work we developed cellular automata that produce similar predictions to the differential equation models, while preserving the rapid modeling and hypothesis testing of cellular automata. Similar models can also be applicable to group animal behavior [11, 16, 19,23]. Our method for deriving rules for cellular automata from observed data in organism growth patterns accounts for soil nutrients, water, root growth patterns, and geology allowing scientists to easily examine the effects of modifying conditions without damaging the environment.

We apply this model to identify factors affecting patterning with respect to growth, die-out, and stabilization in extreme environments. We compare the results of our model with biovermiculation microbial mats growing in acid caves, and cyanobacteria-dominanted desert crust growth in Zzyzx, CA. These models could be used to rapidly check data from space missions to rate the potential of various locations to offer biosignatures.

\section{Cellular Automata}

In the 1940s, John von Neumann developed the first cellular automaton, while working on the self-replicating systems biological problem [31]. Physicist, Conrad Zuse, published a book, Calculating Space, in 1969, that proposed that the universe was the output of a gigantic cellular automaton. In 1970, John Conway developed his Game of Life, which is a two dimensional cellular automaton [13]. In 1983, Stephen Wolfram published the first of many papers on cellular automata culminating in 2002 with the publication of his book, A New Kind of Science [30, 31].

A cellular automaton (CA) is a computational model that is discrete in both space and time. Essentially, we divide space into boxes called cells, and only calculate their values at discrete time using a set of fixed rules. A state could represent any- 
thing, and in our case it represents the amount of water, nutrients and the biomass. The rules describe how the organism grows or dies in the presence of the water, nutrients and competition from other organisms. CA rules are not usually expressed as formulas, rather they are visual, such as drawing pictures of the neighboring cells and then labeling the next state. Cellular automata have been used to study growth and patterns in forests, arid desert environments, predator-prey problems, and sea shells. It has also been used to study areas as diverse as epidemiology and linguistics $[11,16,19,23]$. To avoid tedium, we group similar cell patterns using heuristics discerned from discussions with biologists and born out in practice.

Typically CA with the same starting state are deterministic, we do not use the current value of the state in the calculation of the next state, and use only one state variable. (John von Neumann proposed 29 states in each cell for his self-replicating CA.) Some starting states are so stable that the CA never grows. We consider a generalization of this in which these constraints are relaxed. For this paper, we only relax the determinism constraint, as this allows for more realistic looking results.

As an example we will show a simple CA that is calculated with a radius of 1 . The only values used to calculate the next state are the values in the 8 cells that surround the target cell. The next state of the cell stays the same, unless the sum of the neighbors is 3 (grow) or 7,8 (die).

Fig. 1 First transition of a cellular automaton starting with a square of "living" cells.
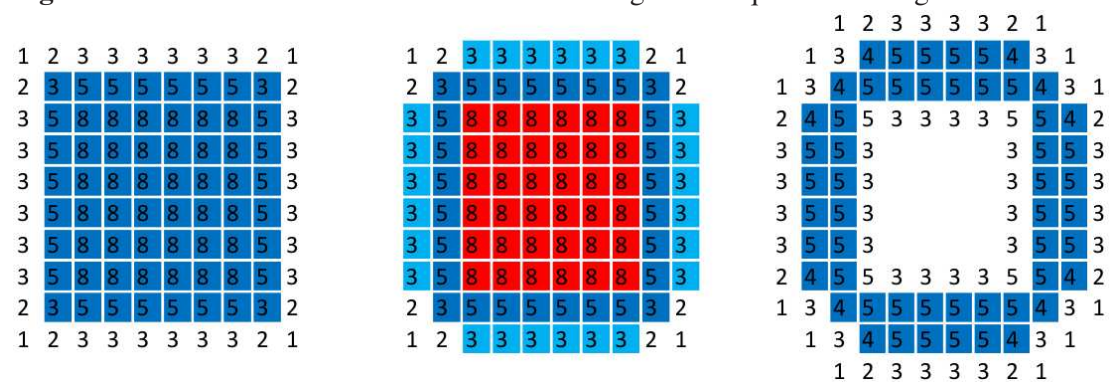

This can be seen in Figure 1, where the initial condition is on the right (starts as the blue square). The numbers are the sum of the neighbors. In the middle figure the cells which are going to die off have been colored red, and the ones about to grow are colored light blue. The final figure on the right shows the next state, with the new neighbor sums. This process would then be repeated.

\section{Cellular Automata and Partial Differential Equations}

Various methods have been researched for directly transforming spatial partial differential equations into cellular automata models through approximation techniques [28] [27]. In Section 3.1, two methods of transforming a general partial differential 
equation into a cellular automata rule are shown. In Section 3.2 the methods are then analyzed using the Z-transform to find the theoretical constraints of stability. Section 3.3 illustrates convergence maps of the cellular automata models created from multiple simulation runs. From this information a set of guidelines was created that can be used to create faster simulations that convergence to stable values.

\subsection{Transformation Using Euler's Methods}

Two methods were developed for directly transforming differential equations into cellular automata. First a generalized partial differential equation was derived from surveying biological partial differential equations such as desert vegetation patterns (Eqs. 1 and 2) [21] [14],

$$
\begin{aligned}
& \frac{\partial n}{\partial t}=\frac{y w}{1+\sigma w} n-n^{2}-\mu n+\nabla^{2} n \\
& \frac{\partial w}{\partial t}=p-(1-\rho n) w-w^{2} n+\delta \nabla^{2}(w-\beta n)-v \frac{\partial(w-\alpha n)}{\partial x}
\end{aligned}
$$

Fick's law on population density (Eq. 3) [26],

$$
\frac{\partial P}{\partial t}=f(t, x, P)+d \nabla_{x}^{2} P
$$

and predator prey models [24]. The following is the general differential equation derived:

$$
f\left(u_{i, j}\right)=\frac{\partial u}{\partial t}=m\left(u_{i, j}\right)+\nabla_{x}^{2} n\left(u_{i, j}\right)+\nabla_{x} o\left(u_{i, j}\right)
$$

In this equation, $u$ will be the values that are simulated. The subscript $i$ is the time index of $u$ and $j$ is the space index. Most of the surveyed differential equations could then be broken up into three groupings of terms based upon whether the Laplacian or gradient operator was applied to them. The terms can be substituted into the $m\left(u_{i, j}\right)$, $n\left(u_{i, j}\right)$, and $o\left(u_{i, j}\right)$ functions.

Once a differential equation is described in this general form, it can easily be transformed into a cellular automata by two different methods. The three point formula is first applied to the general form eliminating the $\nabla_{x}^{2}$ and $\nabla_{x}$ operators. The Forward Euler's method, which is the simpler of the two methods used, can then be applied producing the following formula:

$$
\begin{aligned}
u_{i+1, j}= & u_{i, j}+h_{t}\left(m\left(u_{i, j}\right)+\frac{n\left(u_{i, j+1}\right)-2 n\left(u_{i, j}\right)+n\left(u_{i, j-1}\right)}{h_{x}^{2}}+\right. \\
& \left.\frac{o\left(u_{i, j+1}\right)-o\left(u_{i, j-1}\right)}{2 h_{x}}\right)
\end{aligned}
$$


The Backward Euler's method, which is more stable than the Forward Euler's method, can be applied instead giving the formula:

$$
\begin{aligned}
u_{i+1, j}= & u_{i, j}+\frac{h_{t}}{1-\left.h_{t} \frac{\partial m(u)}{\partial u}\right|_{i, j}}\left(m\left(u_{i, j}\right)+\frac{n\left(u_{i, j+1}\right)-2 n\left(u_{i, j}\right)+n\left(u_{i, j-1}\right)}{h_{x}^{2}}+\right. \\
& \left.\frac{o\left(u_{i, j+1}\right)-o\left(u_{i, j-1}\right)}{2 h_{x}}\right)
\end{aligned}
$$

Equations 5 and 6 are in fact cellular automata rules because they state that the value of $u$ at space $j$ for the next time period is equal to $u$ 's current value plus or minus some factors of its neighboring values $u_{i, j+1}$ and $u_{i, j-1}$. It is now simple to transform a general differential equation into a cellular automata rule by substituting $m\left(u_{i, j}\right)$, $n\left(u_{i, j}\right)$, and $o\left(u_{i, j}\right)$ in the general differential equation form into either the Forward or Backward formulas. It should also be noted that these equations contain two new important variables as the result of the approximations. The variable $h_{x}$ is the step size for space and $h_{t}$ is the step size for time.

\subsection{Theoretical Stability Constraints}

Equations 5 and 6 were analyzed using the Z-transform to create constraints on stability. The analysis was done on a subset of the general form that used linear terms. The following equation was used to represent this general linear form:

$$
f(u)=a_{1} u+b_{1}+\nabla_{x}^{2}\left(a_{2} u\right)+\nabla_{x}\left(a_{3} u\right)
$$

One can assign parts of the equations to the following functions of Equation 4: $m(u)=a_{1} u+b_{1}, n(u)=a_{2} u$, and $o(u)=a_{3} u$. Here the $a$ and $b$ terms are simply coefficients. After the Z-transform was used on Forward and Backward Euler transformed versions of Equation 7, the equation was solved for $U_{j}$. The $i$ index is eliminated in the Z-transform. According to Z-transform theory, if the pole and zero values of $z$ are within the unit circle, then the equation should remain stable. Therefore, constraints of stability can be found by setting the poles and zeros values to less than one. The zero constraint created was the same for both the Forward and Backward Euler's formulas:

$$
1>\left|\frac{-1}{2 b_{1} h_{x}^{2}}\left(\left(2 a_{2}-a_{3} h_{x}\right)\left(U_{j-1}\right)+\left(2 a_{2}+a_{3} h_{x}\right)\left(U_{j+1}\right)\right)\right|
$$

The poles constraint for the Forward Euler's was:

$$
1>\left|1+a_{1} h_{t}-\frac{2 a_{2} h_{t}}{h_{x}^{2}}\right|
$$


The poles constraint for the Backward Euler's was slightly different due to the dividing factor that is part of the Backward Euler's equation:

$$
1>\left|1+\frac{a_{1} h_{t}}{1-a_{1} h_{t}}-\frac{2 a_{2} h_{t}}{\left(1-a_{1} h_{t}\right) h_{x}^{2}}\right|
$$

The pole constraints demonstrate that there needs to be a balancing between $h_{t}$ and $h_{x}$. If one is too big relative to the other term, the right hand side would become larger than one, which would mean the associated equation, either 5 or 6 were unstable.

\subsection{Convergence Maps and Optimum Convergence}

Simulations were run for the Forward and Backward Euler's formulas using Scilab and a one dimensional cellular automata. The results were combined and graphed onto convergence maps shown in Figure 2. In this figure simulations were plotted based upon the $h_{x}$ and $h_{t}$ values used for the Backward Euler's equation. A blue dot represents a simulation that ends in convergence, a green dot represents a simulation that ended in divergence, and a red dot is a simulation that hit the maximum number of iterations allotted without ending in convergence or divergence.

Both the Forward and Backward Euler's convergence maps had a small stripe of convergence in the lower left area. The Backward Euler's map also had an additional area of convergence in the top right, but this area appeared to be an artifact of the Backward Euler's dividing factor. The striped area of convergence was of interest because the convergence values are nearly the same, given the same $h_{x}$ value, even with different $h_{t}$ values. In other words, the manner in which space is discretized affects the outcome of the convergence values but how time is discretized does not.

The convergence map in Figure 2 also shows the pole constraints graphed in black. Through multiple simulations it was found that the area of convergence closely matched the area created by the pole constraints, but not exactly. The difference between the actual area of convergence and the pole constraints appeared to be a constant. Tests also showed that the bottom of the area of convergence converged about ten times faster than the top, therefore making it desirable to use $h_{x}$ and $h_{t}$ values close to the bottom pole constraint, but within the area of convergence.

The convergence map in Figure 3 contains modified zero constraints in magenta. These constraints required substitutions to be made for the $U_{j+1}$ and $U_{j-1}$ terms, which are variables and therefore could not be graphed in the convergence maps. Simulations showed that most of the time that the intersection of the lower pole constraint with the upper zero constraint could be used as an approximation for the maximum $h_{t}$ value for convergence. This information led to a set of guidelines that used the intersection for finding the maximum $h_{t}$ value. A lesser $h_{t}$ value could then be used in the lower pole constraint formula to find an $h_{x}$ value. This would produce an $h_{x}$ and $h_{t}$ pair that would converge quickly because it was a simulation at the 

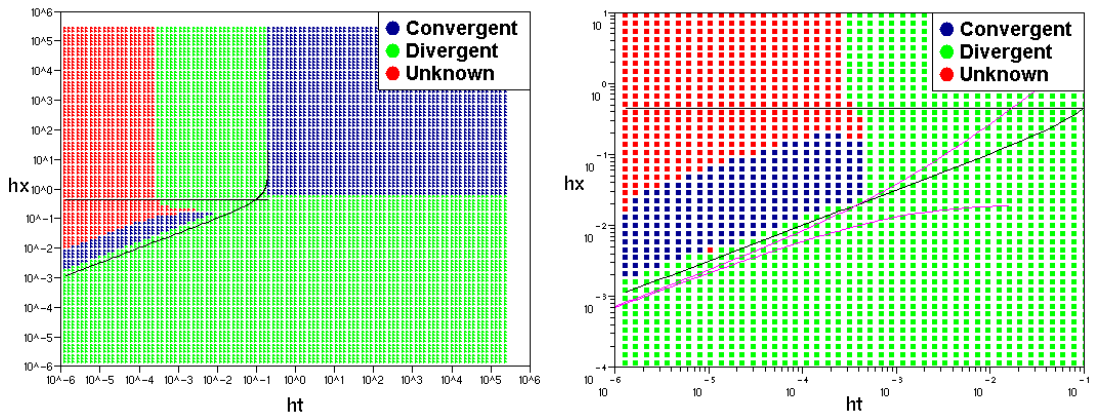

Fig. 2 Convergence map is composed of Fig. 3 Convergence map for the Backward 10,000 simulations with varying $h_{t}$ and $h_{x}$ val- Euler's function shows the pole constraints in ues for the Backward Euler's function. The black and the modified zeros constraints in black lines represent the pole constraints. magenta.

bottom of the area of convergence. Hopefully the direct transformation methods and convergence guidelines can eventually be used in a software tool that will help preform pattern simulations for biologists.

\section{Extremophiles}

Some organisms form patterns based on a combination of intrinsic growth geometries [18], potentially other biological properties [3], and the environmental conditions in which they exist. It has been suggested that there may be significant benefit in such collective community-level behavior and that such organism aggregations behave with a remarkable degree of plasticity and adaptability that allows them enhanced capability to meet changing and challenging growth conditions [20]. Obviously such plasticity with respect to the environment would be particularly pertinent to extremophile organisms living in an array of especially challenging physical and chemical conditions.

The CA models are adapted to the biological and geological conditions found on Earth that are most likely to match those in extreme environments from Earth to Mars to Europa. Indeed, if such biopatterning is as universal as we suspect, it could probably work on any life-bearing planet in the galaxy. As test case models of astrobiological significance, cyanobacteria in desert crusts and biovermiculations (hieroglyphic-patterned microbial mats on cave walls) have been identified. This has grown from our group's participation in NASA's Spaceward Bound Project, a teacher science outreach effort that involves extremophile environment research across a number of scientific and engineering disciplines [10]. 


\subsection{Cyanobacteria}

Cyanobacteria are photosynthetic bacteria relatives that live in a staggering variety of extreme and non-extreme environments across the planet [25]. They are notable for many reasons, including probably being the oldest fossils, the presumptive original producers of free atmospheric oxygen on Earth, the source of much of our oil, and an ability to grow in an amazing array of extreme environments (including Antarctica). The cyanobacteria in extensive desert crusts at Zzyzx Research Station in the Mojave Desert National Preserve, CA, display the structured growth we are considering. Given their pivotal and early role on the Earth, similar organisms are conceivable on early Mars, and their identification is important for biological objectives on Mars and back into the antique history of Earth.

Figure 4 shows a typical cyanobacterially dominated desert crust (aka cryptogamic crust). These patterns are fairly dense, so we used a neighborhood with a range of 1 square ( 1 square in all directions for a total of 8 neighbors). Cyanobacteria often grow in blooms when conditions become favorable as we have demonstrated with watering experiments on these aridland, water-limited communities (P. Boston, unpub. results), so we have modeled this using growth on having 3 neighbors only. We also allowed random death with probability of $10 \%$. The resulting simulation is shown on the right in 4 , and shows much of the same structure as is seen in the picture on left in Figure 4.

Fig. 4 Left Picture is of cyanobacteria-rich desert crust patterns from Zzyzx, CA. Right graphics are simulated cyanobacteria at $t=5$ (Top) and $t=40$ (Bottom).
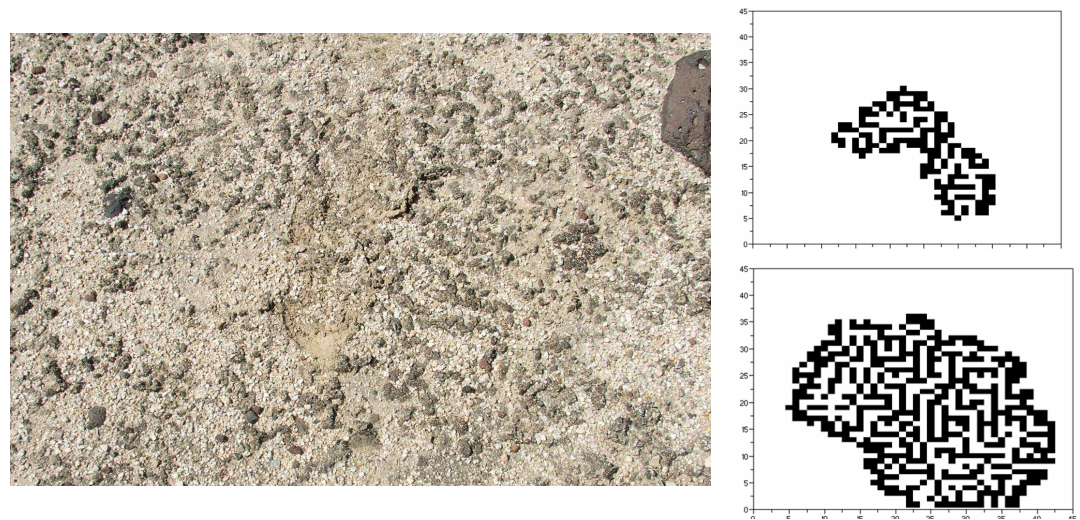


\subsection{Biovermiculations}

Biovermiculations are microbial mats composed of bacteria, extracellular polysaccharide slime, embedded clay and other particles, in situ precipitated minerals (e.g. sulfur, gypsum, carbonate), and even some small invertebrates like mites and nematodes. They exist in a wide variety of chemical and physical subsurface settings including caves, mines, lavatubes, and even human structures. Most recently we have identified a photosynthetic variety on the undersides of translucent rocks in the central Australian deserts (P. Boston, unpub. results).Investigators identified them originally from sulfuric acid caves, see [17]. More recently, observers have begun to see them in a wide variety of chemical and physical subsurface settings including acidic mines, cold carbonate caves, both tropical and alpine lavatubes, and even Mayan ruins.

These structures are interesting because of their intrinsically intriguing biology and geochemistry, the distinctive growth patterns that they exhibit, and also because they may be a highly distinctive biosignature that could be interpretable in extraterrestrial settings based on gross morphology [7]. Interest in the biovermiculations has grown as better methods of studying such structures has become available. They also provide a model system of a biomat that might occur on the interiors of various cave types on Mars. Lavatubes have been identified on Mars [4], and more recently confirmed in a more elaborate study [12]. Mechanisms to create solutional caves in evaporite mineral deposits on Mars have also been proposed [5]. Such potential subsurface habitats could conceivably house or have housed microbial populations on Mars and left traces similar to those found in geomicrobiological communities in Earth's subsurface.

Fig. 5 Left picture is biovermiculation with discontinuities. Right graphic is simulated biovermiculation growth.
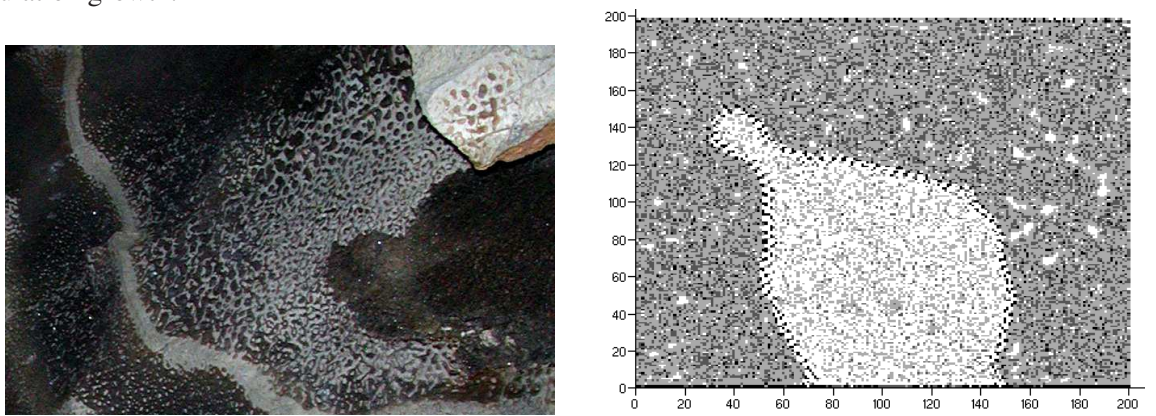

The patterning of biovermiculation growth is still a mystery. To understand it will require simulations that test different sets of rules enabling us to arrive at a good pattern match for the microbial mat growth that we are observing in nature. Such simulations will then be correlated with actual pattern examples from cave 
walls and other occurrences. Figure 5 shows an area that has biovermiculations so thick that they become the solid mat, but there are weird uninhabited areas that follow the rock curvatures. These are not simple water pathways that have prevented growth, so their origin is unknown. In figure 5, no nutrient or water differentiation was induced, only crowding rules, and an ability to pull nutrients from surrounding cells. The result was the depleted region in the middle. Note the shape has many indents, like the actual system in Figure 5.

\section{Identifying Rules from Pictures}

Cellular automata use the neighboring cells (within some radius of effect) to determine the next state of the central cell. If we assume the pattern has been around for a long time, and thus has settled into a relatively stable pattern, then we can assume that the next state of any cell is the same as its current state. This implies that most of the rules can be determined for a stable pattern from a picture by making histograms of the number of neighbors around live and dead centers. We say most because

- relatively stable still implies there will be some cells which will change state in violation of the fundamental assumption,

- not every rule might be active in the picture,

- the scale of the cells must still be determined,

- the radius of effect must still be determined,

- the effects of uneven nutrient distribution is unaccounted for,

- randomness is unaccounted for.

Even with these serious issues, a very good first approximation of the rules can be readily obtained by use of histograms on each cell's neighborhood. The histogram of the live centers (center has value of 1), give us the stay alive rules, see Figure 6. The growing rules can also be seen on the graphs where there are live centers but no dead centers (center has value of 0 ), as the growth rules turns dead centers to live it is theoretically the mechanism that caused this, see Figure 6. In practical histograms, either the left or right side of the ideal histogram will be clear, but the other will be malformed or missing due to which rules were active in the system at that time. A predominantly increasing cellular automaton will have a good right side, while a predominantly decreasing one will have a clear left side.

Consider the graphic of the cellular automaton's state in Figure 7. It is not obvious what the rules were that generated it by simply looking at the picture, other than the radius of effect is likely to be the thickness of a typical line, which is 3 in this case. Indeed the radius of effect for this cellular automaton was 3. Now examine its histogram in Figure 8, which was calculated for neighborhoods of radius 3. The overcrowding limit (upper range of the stay alive rules) should lie between the cross over of the dead center and live center histogram lines and the end of the non-zero portion of the live center histogram, in this case between 34-38. The actual upper limit was 36 , dead center in the range. The growth range should include the region 


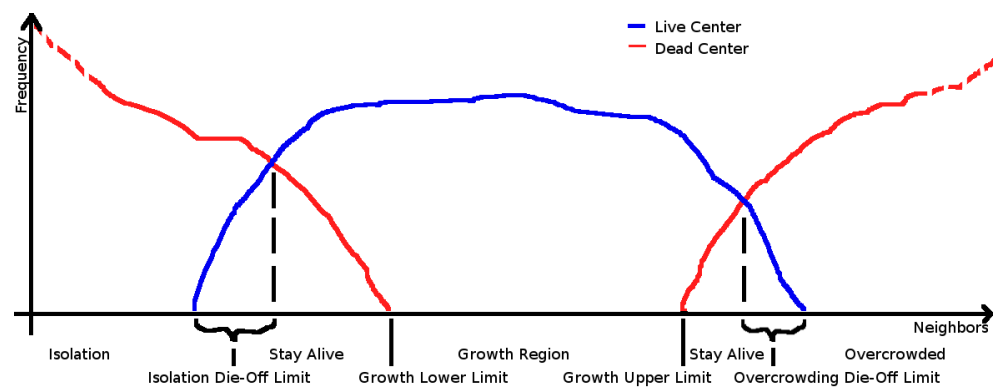

Fig. 6 Idealized histograms of the number of neighbors for both living and dead centers, which shows how to read cellular automaton rules.

where the live center histogram is non-zero but the dead center one is not. It can extend below this, but not above. In this case it should include the region 15-26, perhaps a bit lower. The actual growth region was 13-24, again we have a very good approximation. The isolation die-off limit (lower range of the stay alive rules) will be very hard to determine for this cellular automaton, because the state in the picture is fairly dense and thus there are not many of the lower end of the survival spectrum to determine that limit in this case.

Fig. 7 This graphic is the state of a cellular automaton on a 100 by 100 grid, after 100 time cycles. The cellular automaton used a radius of 3 cells, the center grew if the neighbors numbered 1324 , it kept its state if the neighbors numbered $8-36$, in any other case it died. The initial condition was a box of cells in the center.

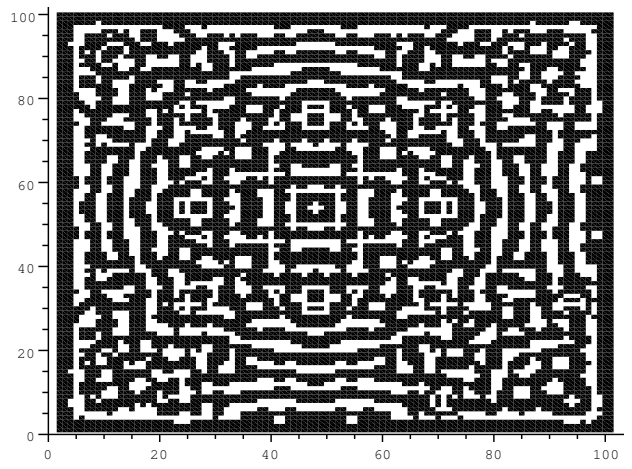

The method is not fool proof, but it does provide a good first approximation of the rules from a picture, and gets very close to most of the four salient numeric quantifiers.

\section{Conclusions and Future Work}

The patterns of microbial extremophile growth, developed using cellular automata, can provide starting templates for the types of patterns we may see in lavatubes and caves on Mars, other planets, and in Earth's own rock record of life. These patterns could provide indicators of life similar to the patterns that indicated water 


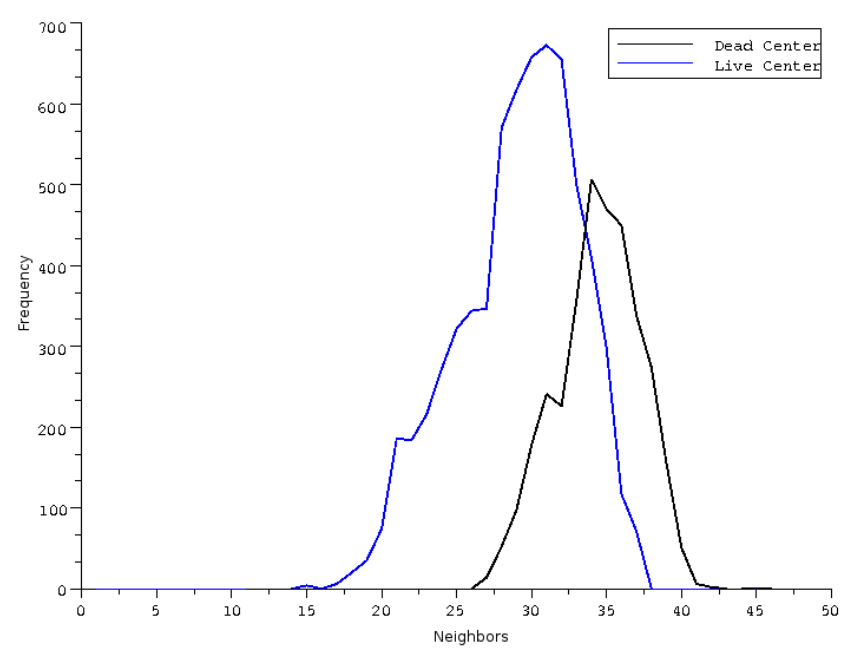

Fig. 8 Dead center and live center histograms of the cellular automaton in Figure 7.

flow and possible "ponds" on the surface of Mars. These templates and the ability to rapidly identify high probability areas for detecting life will be vital to planning future Mars missions. The ability to determine correct simulation parameters is crucial to successfully utilizing these models, and thus the optimal step parameters and histogram rule extraction technique is extremely important. We are continuing our work on both the histogram techniques and biological modeling and validation. In particular, we are working on an automated rule generator for field photographs, as well as both field and lab tests of the validity of the cellular automata models with NASA Ames Research Center colleagues.

\section{References}

1. Catena vol. 37 (1999). Special issue on banded vegetation.

2. Press release images: Spirit. http://marsrovers.nasa.gov/gallery/press/spirit/20050819a.html (2005)

3. Bassler, B.L.: Small talk: cell-to-cell communication in bacteria. Cell (109), 421-424 (2002)

4. Boston, P.: Encyclopedia of Cave and Karst Science, chap. Extraterrestrial Caves, pp. 355358. Fitzroy-Dearborn Publishers, Ltd., London, UK (2004)

5. Boston, P., Hose, L., Northup, D., Spilde, M.: The microbial communities of sulfur caves: A newly appreciated geologically driven system on Earth and potential model for Mars. In: R. Harmon (ed.) Karst Geomorphology, Hydrology, \& Geochemistry, pp. 331-344. Geological Soc. Amer. (2006)

6. Boston, P., Ivanov, M., McKay, C.: On the possibility of chemosynthetic ecosystems in subsurface habitats on mars. Icarus (95), 300-308 (1992) 
7. Boston, P., Spilde, M., Northup, D., Melim, L., Soroka, D., Kleina, L., Lavoie, K., Hose, L., Mallory, L., Dahm, C., Crossey, L., Schelble, R.: Cave biosignature suites: Microbes, minerals and mars. Astrobiology Journal 1(1), 25-55 (2001)

8. CA, K.: Regular and irregular patterns in semiarid vegetation. Science (284), 1826-1828 (1999)

9. Cady, S.: Formation and preservation of bona fide microfossils. In: Signs of Life: A Report Based on the April 2000 Workshop on Life Detection Techniques, Committee on the Origins and Evolution of Life, pp. 149-155. National Research Council, The National Academies Press, Washington, DC (2001)

10. Conrad, L.: Spaceward bound project. website http://quest.nasa.gov/projects/spacewardbound/ (2009)

11. Couzin, I., Krause, J., James, R., Ruxton, G., Franks, N.: Collective memory and spatial sorting in animal groups. J. Theor. Biol. (218), 1-11 (2002)

12. Cushing, G., Titus, T., Wynne, J., Christensen, P.: Themis observes possible cave skylights on mars. Geophysical Research Letters 34(L17201) (2007)

13. Gardner, M.: The fantastic combinations of john conway's new solitaire game 'life'. Scientific American 223, 120-123 (1970)

14. von Hardenberg, J., Meron, E., Shachak, M., Zarmi, Y.: Diversity of vegetation patterns and desertifcation. Phys Rev Lett (87:198101-14) (2001)

15. HilleRisLambers, R., Rietkerk, M., van den Bosch, F., Prins, H., de Kroon, H.: Vegetation pattern formation in semi-arid grazing systems. Ecology (82), 50-62 (2001)

16. Hoare, D., Couzin, I., Godin, J.G., Krause, J.: Context-dependent group size choice in fish. Animal Behavior 67, 155-164 (2004)

17. Hose, L., Palmer, A., Palmer, M., Northup, D., Boston, P., Duchene, H.: Microbiology and geochemistry in a hydrogen sulphide-rich karst environment. Chemical Geology 169, 399$423(2000)$

18. Jacob, E.B., Aharonov, Y., Shapira, Y.: Bacteria harnessing complexity. Biofilms (1), 239-263 (2004)

19. Krause, J., Tegeder, R.: The mechanism of aggregation behavior in fish shoals: individuals minimize approach time to neighbours. Anim. Behav. (48), 353-359 (1994)

20. Levine, H., Jacob, E.B.: Physical schemata underlying biological pattern formation-examples, issues and strategies. Journal of Physical Biology (1), 14-22 (2004)

21. Meron, E., Gilad, E., von Hardenberg, J., Shachak, M., Zarmi, Y.: Vegetation patterns along a rainfall gradient. Chaos, Solutions and Fractals (19), 367-376 (2004)

22. Nealson, K., Conrad, P.: Life: past, present and future. Phil. Transact. Royal Soc. London Ser. B-Biol. Sci. 354(1392), 1923-1939 (1999)

23. Pitcher, T., Misund, O., Fernö, A., Totland, B., Melle, V.: Adaptive behaviour of herring schools in the norwegian sea as revealed by high-resolution sonar. ICES Journal of Marine Science (53), 449-452 (1996)

24. Savill, N.J., Hogeweg, P.: Competition and dispersal in predator-prey waves. Theoretical Population Biology 56, 243-263 (1999)

25. Seckbach, J.: Cellular Origin, Life in Extreme Habitats and Astrobiology, chap. Algae and Cyanobacteria in Extreme Environments, p. 814. Springer, Dordrecht, Netherlands (2007)

26. Shi, J.: Partial differential equations and mathematical biology. http://www.resnet.wm.edu/ jxshix/math490/lecture-chap1.pdf (2008)

27. Strader, B.: Simulating spatial partial differential equations with cellular automata. M. cs., CSU San Bernardino, San Bernardino, CA, USA (2008)

28. Strader, B., Schubert, K., Gomez, E., Curnutt, J., Boston, P.: Simulating spatial partial differential equations with cellular automata. In: H.R. Arabnia, M.Q. Yang (eds.) Proceedings of the 2009 International Conference on Bioinformatics and Computational Biology, vol. 2, pp. 503-509 (2009)

29. Thiéry, J., d'Herbès, J., Valentin, C.: A model simulating the genesis of banded vegetation patterns in niger. J. Ecol. (83), 497-507 (1995)

30. Wolfram, S.: Twenty problems in the theory of cellular automata. Physica Scripta (T9), 170$183(1985)$

31. Wolfram, S.: A New Kind of Science. Wolfram Media Inc. (2002) 